\title{
Credit, debit \& funding value adjustments en valoración de instrumentos financieros: la realidad económica detrás del ajuste contable*
}

doi:10.11144/Javeriana.cc17-44.cdfv

\section{Pablo Martínez}

Contador público. Director de políticas contables, Colpa-

tria Multibanca, del grupo Scotiabank.

Correo electrónico: pabwmabi@gmail.com 
Resumen Este documento pretende resaltar la creciente importancia que para la profesión contable tienen el conocimiento y el continuo aprendizaje de conceptos profundos de finanzas y riesgos. Mediante un análisis económico-contable de los ajustes por riesgo de crédito de contraparte y propio propuestos por la IFRS 13, el autor pone de manifiesto debilidades recurrentes en los procesos de emisión y aplicación de normas contables que deben ser atendidos como desafíos propios de la profesión a corto y mediano plazo para garantizar que no se desvirtúe la esencia económica y financiera de la contabilidad. Se concluye que tener estándares políticamente fuertes pero técnicamente mal elaborados es servir a intereses diferentes a los financieros y para la toma de decisiones. La contabilidad debe imitar la complejidad de las transacciones y no ocultar realidades como aparente solución a los problemas.

\section{Palabras clave Ajuste por riesgo de crédito; ajuste} por riesgo de débito; ajuste del valor de financiamiento; valor justo; riesgo de contrapartida; crédito; riesgo; riesgo crediticio; NIIF

\section{Códigos JEL C52, M41, P34}

\section{Credit, Debit \& Funding Value}

\section{Adjustments in the Valuation of Financial} Instruments: The Economic Reality

\section{Behind Accounting Adjustment}

\footnotetext{
Abstract This document aims to highlight the growing importance of knowledge and continuous learning of profound concepts of finance and risk for the accounting profession. By means of an economic-accounting analysis of the counterparty credit risk and own credit risk adjustments proposed by IFRS 13, the author reveals recurrent weaknesses in the issuance and application processes of accounting standards that must be addressed in the short and medium term as challenges characteristic of the profession to ensure that the economic and financial essence of accounting is not distorted. We conclude that having politically strong but
}

technically poorly elaborated standards is to serve interests different to financial and decision-making ones. Accounting should mimic the complexity of transactions and not hide realities as an apparent solution to problems.

Keywords credit value adjustment; debit value adjustment; funding value adjustment; fair value; counterparty risk; credit; risk; lending risk; IFRS

\section{Credit, debit \& funding value adjustments em valoração de instrumentos financeiros: a realidade económica por trás do ajuste contábil}

Resumo Este documento objetiva destacar a crescente importância que para a profissão contábil tem o conhecimento e a continua aprendizagem de conceitos profundos de finanças e risco. Mediante análise económico-contábil dos ajustes por risco de crédito de contraparte e próprio sugeridos pela IFRS 13, o autor põe de manifesto fraquezas recorrentes nos processos de emissão e aplicação de normas contábeis que devem ser atendidos como desafios próprios da profissão a corto e médio prazo para garantir que não se desvirtue a essência económica e financeira da contabilidade. Conclui-se que ter padrões politicamente fortes pero tecnicamente mal elaborados é servir para interesses diferentes aos financeiros e para a tomada de decisões. A contabilidade deve imitar a complexidade das transações e não ocultar realidades como aparente solução aos problemas.

Palavras-chave Ajuste do valor de crédito; ajuste do valor de débito; ajuste a valor de financiamento; valor justo; contraparte de risco; crédito; risco; risco de crédito; IFRS

\section{Breve historia y contextualización}

Los cambios relativamente recientes en los requerimientos contables han hecho que diversas 
instituciones y cuerpos alrededor del mundo debatan y estudien a profundidad las implicaciones que las nuevas formas de contabilizar traen a las entidades tanto desde el punto de vista del preparador, como del tomador de decisiones. A pesar de que ha existido una creciente aceptación por parte de los cuerpos regulatorios y los emisores de estándares en los últimos años, las mediciones a valor razonable permanecen aún hoy como uno de los mayores problemas en la práctica contable.

Muchos se equivocan cuando califican el valor razonable como un concepto nuevo. Esta percepción generalizada ha aumentado el debate y las críticas, lo que provoca que ante el desconocimiento de ciertos eventos/debates históricos, estemos "condenados" a repetirlos. Por ejemplo, la técnica de valorar a mercado fue utilizada ampliamente a comienzos del siglo XX durante la Gran Depresión [1929-1939] y hasta su extinción hacia 1940, cuando fue abolido por completo el uso del llamado markto-market accounting (MtM) en las valoraciones de balance. La razón principal fue que era causante de la severidad de las crisis (algo similar a lo que se argumentó en la más reciente crisis hipotecaria).

Para esa época, la contabilidad centrada en costos históricos - mucho más reciente que la centrada en exit prices - fue reemplazada al ser considerada mucho más conservadora. Sin embargo, a mediados de los años 80, la innovadora contabilidad centrada ahora en costos enfrentó importantes desafíos (Martínez-Pineda, 2010, p. 79). A manera de ejemplo, los movimientos en el mercado provocados por las fluctuaciones de las tasas de interés, no eran re- conocidos en los estados financieros. Ahorros y préstamos debían pagar un mayor interés por causa de inflaciones altas que no compensaban los ingresos por intereses generados por créditos hipotecarios a tipo fijo y largo plazo. En ese orden de ideas, el MtM de los activos de los bancos no compensaba el desnivel en sus pasivos a tipo variable, lo que hacía muchas instituciones económicamente insolventes ( $\sin$ embargo, estas pérdidas no se registraban en los balances).

La contabilidad centrada en costos históricos ocultó pérdidas potenciales, lo que permitió que diversos principios contables fuesen violados (por ejemplo, negocio en marcha) y, lo peor, que avanzasen sin ser detectados.

Posteriormente, por los años 80, el entorno económico y de negocios cambió, para dar origen a la creación de instrumentos financieros nuevos (debido a la desregulación de los tipos de interés). Diversos pronunciamientos comenzaron a aplicar mediciones basadas en el mercado de forma dispersa, hasta 2006, cuando la Financial Accounting Standard Board, FASB, emitió el SFAS 157 para proporcionar guías adecuadas y unificadas sobre cómo medir el valor razonable de activos y pasivos (FASB, 2006).

Por aquellas fechas, mientras se desarrollaba la crisis financiera mundial, muchos bancos e instituciones financieras que llevaban mediciones a valor razonable en sus balances, registraron grandes montos de pérdidas no realizadas. Para garantizar índices apropiados de capital mínimo requerido, fueron presionadas a vender activos no financieros, lo que acentúo aún más el carácter sistémico de la crisis. Producto de esta situación, la estadounidense 
Security and Exchange Commission (SEC) emitió el Report and Recommendations Pursuant to Section 133 of the Emergency Economic Stabilizing Act of 2008: Study on Mark-to-Market Accounting (SEC, 2008). En este reporte, la entidad reafirma la solidez de los reportes basados en valores razonables concluyendo que "la crisis es principalmente resultado de malas decisiones de operación, inversión y financiación, pobre administración del riesgo y en algunas circunstancias, fraude" (Ryan, 2008).

En este sentido, la crisis - especialmente la del crédito- puso de manifiesto diversos problemas relacionados con la administración de riesgos financieros que, a su vez, impactaron de forma importante la valoración de los instrumentos financieros en los balances de las entidades. En la definición de valor razonable, un comprador/vendedor debidamente informado tendría en cuenta todos los riesgos a la hora de entrar en una transacción de compra (activos) o de transferencia (pasivos). Uno de los más representativos, y sobre el cual las normas contables hacen énfasis, es el riesgo de incumplimiento $^{1}$ (incluye el riesgo de crédito) en la medición del valor razonable.

La incorporación del riesgo de incumplimiento - en especial, el riesgo de crédito-es un paso fundamental en la medición a valor razonable.

FASB (2008) lo resume así:

...Conceptually, credit standing is an essential component of a fair value measurement. A measurement that does not consider the

1 Non-performance risk, en inglés. effect of [an] entity's credit standing is not a fair value measurement.

Regardless of the valuation technique used, an entity must include appropriate risk adjustments that market participants would make for nonperformance and liquidity risks.

[... Conceptualmente, la solvencia crediticia es un componente esencial de las mediciones a valor razonable. Una medición que no considere el efecto de la solvencia crediticia de una entidad no es una medición a valor razonable].

\section{Riesgo de crédito y riesgo de contraparte: definición}

Para analizar con más detalle el requerimiento contable y delimitar el objeto de estudio, es preciso tener claridad conceptual sobre algunas definiciones que parecen similares, pero en realidad son aplicables a diferentes instrumentos financieros y no deben confundirse entre sí cuando se incorpora el ajuste por valoración.

Aunque similares en naturaleza, no debe confundirse el riesgo de préstamo (lending risk) con el riesgo de contraparte (counterparty risk). Tradicionalmente, al riesgo de crédito (credit risk) se le ha atribuido un concepto similar al riesgo de préstamo. Esto es aplicable cuando una parte (A) debe principal + intereses (es decir, un instrumento de deuda) a otra parte (suponga, Bancobertura) y podría incumplir en el pago de alguno, otro o ambos por motivo de insolvencia. Esto puede ser aplicado a préstamos, bonos, créditos etc., Sin embargo, el concepto de riesgo de crédito ha evoluciona- 
do para abarcar otros riesgos diferentes al de préstamo.

El riesgo de préstamo se caracteriza fundamentalmente por:

- El monto adeudado o por cobrar (ejemplo, principal, interés) durante el período del préstamo es conocido con un grado razonablemente aceptable de certeza. Variables de mercado ajenas a la valoración como tipos de interés crean solo una incertidumbre moderada sobre cualquier cuota contingente. Por ejemplo, un bono a tipo fijo con un valor a la par de $\$ 1.000$, el monto nocional al riesgo por la vida del bono es cercano a \$1.000. Los pagos amortizarán el principal, pero se puede predecir con una precisión aceptable el costo amortizado a cualquier fecha de balance.

- Solo una parte asume el riesgo de crédito². El inversionista asumiría la totalidad de la pérdida eventual, pero el emisor del bono no perderá, si el comprador del bono entra en quiebra.

Con el riesgo de contraparte, aunque la causa de pérdida continúa siendo la misma (incumplimientos debido a defaults), dos aspectos diferencian los contratos con riesgo de contraparte y riesgo de crédito:

- El valor del contrato a futuro es incierto. Por ejemplo, el valor razonable de un derivado a una fecha específica $t$ será el valor presente neto de todos los flujos de efecti-

2 Recuerde la definición de instrumento financiero como contrato que tiene dos partes. En el caso presente, el riesgo de préstamo no es bilateral. En otras palabras, solo una parte asume la posible pérdida futura. vo futuros estimados. Este valor puede ser positivo o negativo y en la gran mayoría de los casos, la incertidumbre asociada es significativa.

- Desde que el valor del derivado o el valor a cancelar pueda ser positivo o negativo, el riesgo de contraparte es bilateral (excepto en opciones). En una transacción en la que se usan instrumentos financieros derivados, cada contraparte está expuesta al riesgo con respecto a la otra.

En otras palabras, la característica distintiva del riesgo de contraparte comparado con otros riesgos similares es que el valor del contrato futuro es incierto, tanto en magnitud como en signo.

\subsection{Incorporación del riesgo de crédito en activos financieros}

Respecto a activos financieros, el riesgo de crédito se incorpora independientemente de cómo sean clasificados. Para los activos financieros medidos posteriormente al costo o costo amortizado, hay modelos de pérdidas (incurridas/esperadas) dependiendo del estándar que se esté utilizando.

Para mediciones al valor razonable clasificadas en la jerarquía 1 o 2 y en los que se utilizan técnicas de medición exclusivas de mercado ${ }^{3}$, no es necesario estimar el riesgo de crédito pues se asume que el precio publicado fue establecido por los participantes de mercado teniendo en cuenta hipótesis sobre el riesgo.

Sin embargo, cuando se utilizan técnicas diferentes a las de mercado (por ejemplo, ingreso)

3 Activos o pasivos idénticos o similares. 
dentro de jerarquías 2 y 3 , la entidad debe asegurarse de que los flujos de efectivo o los tipos de descuento incluyan hipótesis sobre el riesgo.

Aunque el cálculo del riesgo de crédito pudiese revestir cierta complejidad, la incorporación en el modelo es relativamente simple cuando la exposición del instrumento financiero no cambia en el tiempo. Una prima por riesgo busca compensar a los participantes de mercado adversos para soportar la incertidumbre inherente a los flujos de efectivo. Las mediciones a valor razonable incluyen estos ajustes que reflejan el importe que los participantes de mercado reclamarían como respuesta a la incertidumbre.

En ese orden de ideas, el factor riesgo en las mediciones a valor razonable que utilizan flujos de caja descontados, puede ser estimado como un ajuste a los tipos de interés de descuento o como un ajuste de la técnica de valor presente esperado.

En todo caso, un ajuste a los flujos de efectivo ponderados por riesgo o un ajuste a los tipos de descuento incluyendo una prima por riesgo, da como resultado un menor valor presente del instrumento financiero registrado en balance y una pérdida en resultados por valoración, lo que es congruente desde el punto de vista económico al reflejar una pérdida no realizada que la entidad estuviese dispuesta a asumir hoy vía disposición del activo financiero.

\subsection{Incorporación del riesgo de crédito en pasivos financieros}

La incorporación del riesgo de crédito en las mediciones a valor razonable de pasivos financieros y derivados siempre ha estado presente como requisito en los estándares. Sin embargo, la NIIF 13.42 lo hace mucho más claro:

El valor razonable de un pasivo refleja el efecto del riesgo de incumplimiento. El riesgo de incumplimiento incluye, pero puede no limitarse, al riesgo de crédito propio de una entidad (como se define en la NIIF 7 Instrumentos Financieros: Información a revelar). El riesgo de incumplimiento se supone que es el mismo antes y después de la transferencia del pasivo.

Con relación a la valoración a partir del día 1 , si el pasivo financiero se mide a valor razonable, se consideran todos los factores que afectan los cambios en su valor, incluido el riesgo de crédito propio de la entidad que emite el pasivo financiero.

En pasivos financieros con riesgo de préstamo (y no de contraparte), la empresa emisora valora el instrumento financiero como lo valoraría la empresa que lo mantiene como activo (el inversionista $)^{4}$. En ese orden de ideas, deberá considerar la curva libre de riesgo más una prima por riesgo. El efecto anterior supone que los pasivos financieros de la entidad emisora sean reconocidos por un menor valor (al descontar con primas por riesgo) con contrapartida a ingresos por valoración.

\footnotetext{
4 NIIF 13.37: Cuando un precio cotizado para la transferencia de un pasivo idéntico o similar o un instrumento de patrimonio propio de la entidad no está disponible y la partida idéntica se mantiene por otra parte como un activo, una entidad medirá el valor razonable del pasivo o instrumento de patrimonio desde la perspectiva de un participante de mercado que mantiene la partida idéntica como un activo en la fecha de la medición (itálicas añadidas).
} 
Este efecto ha hecho que muchas entidades valoren sus instrumentos financieros pasivos mantenidos a valor razonable e incluyan en la valoración, curvas que incluyen primas por riesgo propias. Esta inclusión, sostienen algunos, crea un efecto contraintuitivo. Quienes critican la medida argumentan que un empeoramiento en la calidad crediticia de la entidad no debería ser reflejado, mediante el reconocimiento de un ingreso en la cuenta de resultados.

Jon Gregory (2012) menciona:

More recently, in 2011 banks have made similar large accounting gains as their credit spreads widened in the midst of the euro crisis and other market turbulence. Articles reporting this have made statements such as "The profits of British banks could be inflated by as the value of their debt" and describing FVA as "counter-intuitive but powerful accounting effect that means banks book a paper profit when their own credit quality declines". Perhaps the most striking illustration of the effect was with UBS, where a "Sfr1.8 billon DVA gain nearly cancelled out the Sfr 1.9 billon it lost in an alleged rogue trading incident".

[Más recientemente, en 2011 los bancos han registrado grandes ganancias contables conforme sus diferenciales de crédito se ampliaron en medio de la crisis del euro y otras turbulencias de mercado. Los artículos que han informado esto han hecho declaraciones como "Las ganancias de los bancos británicos podrían inflarse, debido al valor de su deuda" y describiendo FVA como "contraintuitivo pero poderoso efecto contable que significa que los bancos registren ganancias en el papel cuando su propia calidad del crédito disminuye”. Quizás la ilustración más llamativa del efecto fue UBS, cuya ganancia de "1.800 millones de francos suizos DVA casi cancela los 1.900 millones de francos suizos de pérdida en un supuesto incidente comercial"].

Christopher Whittall (citado por MoralesDíaz, 2010) estableció:

Deutsche Bank registró en 2008 un beneficio de 4.700 millones de euros con relación a los pasivos financieros designados a valor razonable con cambios en la cuenta de pérdidas y ganancias. Durante el año, el coste de la protección a 5 años en el mercado de Credit Default Swaps (CDS) subió de 46 puntos básicos a 134 puntos básicos (Whittall, 2009).

HSBC que reportó un ingreso de 6.600 millones de dólares por cambios en el propio riesgo de crédito en 2008, registró una pérdida de 2.500 millones en el primer trimestre de 2009, cuando el coste de su CDS pasó de 94 a 75 puntos básicos (Whittall, 2009).

Este argumento fue desarrollado por IASB en su documento Credit Risk in Liability Measurement (June 2009, p. 12):

Argument. When liability measurement includes credit risk, an entity reports a gain from a decline in the credit quality of its liabilities. This gain (or loss, in the case of improving credit quality) is counter-intuitive. 
Gains should result from improvements in an entity's financial position, not declines. Reporting a gain from a decline in credit quality is potentially misleading and can mask a deteriorating situation.

[Argumento. Cuando la medición de un pasivo incluye riesgo de crédito, una entidad reporta una ganancia ante una disminución en la calidad crediticia de sus pasivos. Esta ganancia (o pérdida, en el caso de que se mejore la calidad del crédito) es contraintuitiva. Las ganancias deberían resultar de las mejoras en la posición financiera de la entidad, y no del deterioro. Reportar una ganancia de una disminución en la calidad del crédito es potencialmente engañoso y puede ocultar una situación de deterioro].

A pesar de una primera interpretación, varios autores (entre ellos IASB) establecen que el ajuste tiene lógica económica. Reconocer ganancias cuando la calidad del crédito de la entidad emisora se deteriora, subyace a las teorías económicas financieras trabajadas por Robert

C. Merton:

Una empresa tiene dos formas de financiación. A través de deuda (debt) con acreedores y a través de la emisión de acciones (equity). Los acreedores tienen un derecho contractual de cobro y los accionistas tienen una participación residual de los activos, una vez deducidos los pasivos (patrimonio). Suponga una entidad con importes registrados en sus activos que no cambian de valor. En cambio, el valor de sus pasivos varía como consecuencia de modificaciones en el valor presente de sus flujos de efectivo. La ecuación patrimonial es afectada de tal forma que el patrimonio es modificado en un valor proporcional al aumento (disminución) del valor presente de sus pasivos. En otras palabras, bajo supuestos de valor de activos constante, si los pasivos aumentan su valor, el retorno para los accionistas sería menor. Contrariamente, si los pasivos disminuyen de valor, es decir, las exigencias de los acreedores son menores, debido, por ejemplo, a aumentos en las primas por riesgo y liquidez, el retorno para los accionistas aumentaría.

Bajo estas circunstancias, los grupos de interés de la entidad tienen diferentes preferencias a la hora de invertir. Los participantes de mercado preferirán destinar sus recursos en entidades cuyos pasivos tengan menor valor (porque pagan menos intereses). Siguiendo el ejemplo, si la entidad deteriorase su calidad crediticia (y si los pasivos se midiesen a costo amortizado y no se actualizasen ante primas por riesgo), los acreedores (debt holders) se han comprometido a aceptar un interés que en realidad es bajo. Lo anterior conlleva un beneficio para los accionistas cuyas inversiones se valorizarán a comparación con las que obtendría pagando mayores intereses invirtiendo en instrumentos de deuda (pasivos para la entidad).

La contabilidad a valor razonable de los pasivos puede ser vista de dos formas por acreedores y accionistas:

- Una vez los acreedores han concedido un préstamo a la empresa (condiciones normales, como plazo, tipo fijo, cuota fija), las 
condiciones de mercado pueden favorecer a los accionistas. Los tipos de interés libres de riesgo suben. La empresa se financia vía deuda a unos precios más baratos que cualquier participante de mercado al pedir financiación haría. Por consiguiente, el valor de la empresa se incrementa.

- Una vez los acreedores han concedido un préstamo a la empresa (condiciones normales, como plazo, tipo fijo, cuota fija), las condiciones de mercado pueden favorecer a los acreedores. Los tipos de interés de mercado disminuyen. La empresa se ha comprometido a pagar unos intereses mayores a los tipos de interés que hubiera pagado antes. Por consiguiente, el valor de la empresa disminuye.

- Si los acreedores conceden un préstamo a la empresa a tipo de interés variable, generalmente su valor razonable no cambiará de valor; esto supondría que ni los accionistas ni los acreedores se verán beneficiados, precisamente porque la empresa siempre está pagando a tipos de mercado.

\section{Así, la lectura de los pasivos financieros es} contraria a la de los activos. Una empresa se beneficia de un aumento en los activos y contrariamente, de una disminución de sus pasivos. IASB (2012, p. 16) lo establece así:

Argument. Liabilities and equity represent the two classes of claims against the entity. A change in the credit risk of the entity's liabilities represents a transfer of wealth between those two classes. Lenders' interests are usually senior to those of equity holders and their potential gains and losses are bounded by the terms of a contract. Equity holders of an entity are not required to make any additional investment to cover losses incurred by the entity except to the extent that the equity holders have a binding obligation to do so. As the entity's ability to pay its liabilities diminishes, the effect on owners' claims is limited to the amount of their investment. Therefore, the apparent gain to the borrower can be seen as an allocation of claims between the borrower's owners and its lenders.

An entity's statement of financial position includes its recognised assets and two sets of claims against those assets - those of owners and those of others. The value of the claims derives from the value of the assets. This is captured in the classic accounting equation that assets equal liabilities plus equity. Owners' relative share of the claims increase or decrease as the value of the entity's total assets increase or decrease, in the absence of additional borrowing or other new liabilities.

[Argumento. Los pasivos y el patrimonio representan dos clases de reclamaciones contra la entidad. Un cambio en el riesgo de crédito de los pasivos de la entidad representa una transferencia de riqueza entre estas dos clases. Los intereses de los prestamistas suelen ser superiores a los de los accionistas y sus ganancias y pérdidas potenciales están limitadas por los términos de un contrato. Los tenedores de instrumentos de patrimonio de la entidad no están obligados a realizar inversiones adicionales para cubrir pérdidas 
sufridas por la entidad, salvo en la medida en que los accionistas tengan una obligación vinculante de hacerlo. A medida que la capacidad de la entidad para pagar sus pasivos disminuye, el efecto sobre los reclamos de los propietarios se limita al monto de su inversión. Por lo tanto, la ganancia aparente del prestatario puede ser vista como una asignación de reclamos entre prestatarios y sus prestamistas.

El estado de situación financiera de una entidad incluye activos reconocidos y dos conjuntos de reclamaciones contra esos activos, aquellas de los propietarios y las de otros. El valor de las reclamaciones se deriva del valor de los activos. Esto es capturado en la ecuación de contabilidad de activos = pasivos + patrimonio. La participación relativa de los propietarios de los créditos aumenta o disminuye a medida que aumenta o disminuye el valor de los activos totales de la entidad, en ausencia de préstamos adicionales $u$ otros nuevos pasivos].

Más recientemente, la nueva NIIF 9 (versión final) deja en claro que los efectos en los cambios del riesgo de crédito de un pasivo medido a valor razonable no deberían afectar resultados, a menos que sea mantenido para negociar. En otras palabras, aquellos pasivos financieros con designación de valor razonable (fair value option) pueden llevar la afectación por cambios en la prima por riesgo en la medición del valor razonable, a otros resultados integrales si haciéndolo de esa forma, reducirían la asimetría contable que la opción pretende eliminar.
En otras palabras, si la entidad no espera que otro instrumento medido a valor razonable con cambios en resultados (el instrumento de cobertura) se mida a valor razonable, de forma que los efectos de los cambios en el riesgo de crédito del pasivo se compensen con los cambios en el riesgo de crédito del otro instrumento, deberá llevar estos cambios al patrimonio vía otro resultado integral. De otra forma, deberá afectar resultados basados en la relación económica que hay entre las características del pasivo y las del instrumento financiero que sirve como match.

En relación con otros activos financieros medidos a valor razonable (típicamente derivados) y que no fueron designados como coberturas contables calificadas, los cambios en su valor razonable atribuibles al riesgo de contraparte se registran directamente en resultados.

\section{Incorporación del riesgo de crédito en valoración de derivados financieros}

Como lo mencioné con anterioridad, no resulta apropiado hablar indistintamente de riesgo de crédito (credit risk), riesgo de préstamo (lending risk) y riesgo de contraparte (counterparty risk).

Cuando se hace referencia al riesgo de crédito aplicado a derivados, se debe utilizar el término riesgo de contraparte. El entendimiento de este riesgo requiere a su vez una conceptualización de otros riesgos, como el de mercado y el de préstamo.

El riesgo de contraparte se puede dividir en: 1) riesgo de mercado, el cual define la exposición de un activo al momento t (llamada expo- 
sure at default), y 2) riesgo de crédito, al tener que definir la calidad crediticia de la contraparte. Debido a que el riesgo de crédito es compartido por todos los instrumentos financieros, el riesgo de mercado solo es aplicado a derivados al ser la exposición futura incierta a la fecha de cumplimiento $t$ (llamada probability of default).

¿Cómo la contabilidad se apoya en las teorías financieras y económicas para cuantificar e implementar el ajuste por riesgo de contraparte?

Hoy, el tema asusta a muchos contadores y reguladores al punto de querer "sobretercerizar" los cálculos, en lugar de hacer una evaluación juiciosa y reflexiva dentro de la profesión y las academias y promover así una mayor interdisciplinariedad. La solución de algunos organismos reguladores es descargar la responsabilidad en una proveeduría de precios y dejar el componente de juicio y análisis fuera del alcance de la práctica profesional contable.

Limitarse a la operatividad de contabilizar el dato recibido es una injustificada transferencia de responsabilidades hacia un tercero experto. Este punto se expondrá con mayor detalle en las conclusiones.

\subsection{CVA/DVA/FVA - Credit/Debit/ Funding value adjustment}

Los derivados transados OTC que son clasificados dentro de los niveles 2 y 3 en la jerarquía del valor razonable son valorados con modelos. El resultado siempre corresponderá a una estimación o aproximación al valor razonable que, por las características propias de los derivados, no pueden ser medidos con completa certeza. Como resultado, estas valoraciones son ajusta- das para considerar factores que, a su vez, los participantes de mercado tengan en cuenta para establecer el precio del activo/pasivo.

Para estos instrumentos financieros, las entidades normalmente comienzan utilizando inputs de mercado medios mid-market, como curvas interbancarias cupón cero sin tener en cuenta hipótesis sobre el riesgo (es decir, primero se valora el derivado risk-free). Una vez este procedimiento es realizado, cada entidad aplica diferentes ajustes a su proceso valorativo. Dependiendo de su naturaleza, los ajustes aumentan (reducen) el valor razonable del derivado risk-free. Estos ajustes típicamente incluyen ajustes por riesgo de contraparte y propio (CVA/DVA) y ajuste de valor por financiación (FVA).

Para los instrumentos financieros clasificados en nivel 3, los procesos de valoración involucran inputs no observables considerados significativos que no pueden ser corroborados por el mercado. Una medición clasificada dentro del nivel 3 de un activo o pasivo financiero, requiere la estimación de al menos un input que tenga un impacto importante en la valoración. La IFRS 13 no especifica cuándo un input es considerado significativo, pero la práctica de mercado asume que un input es significativo si contribuye entre 5 y 10\% en la valoración del instrumento financiero. Dentro de esta categoría, típicamente se incluyen derivados exóticos como opciones cesta (basket options) o spread para los cuales el precio del (los) subyacente (s) no es observable (por ejemplo, bonos no líquidos, préstamos u otros). Swaps de incumplimiento crediticio (CDS) para los cuales los spread de crédito son no observables, IRS, CCS, 
swaps de inflación, FX forwards y opciones con fechas de cumplimiento aplazadas en el tiempo (2, 3 o más años) pueden ser clasificados también dentro del nivel 3 de los instrumentos financieros.

Similar a lo que sucede con los derivados que son nivel 2, el valor razonable de aquellos clasificados en nivel 3 es calculado ajustando el tipo de interés libre de riesgo, mediante ciertos elementos adicionales que reflejan incertidumbres referentes al proceso de proyección y resultados arrojados por el modelo de valoración risk-free.

\section{Ajustes comprador-vendedor (Adjusts Mid-to-bid and mid-to-ask)}

Como principio de la IFRS 13, cuando un activo/pasivo medido a valor razonable tiene un precio de comprador y vendedor, la entidad utilizará el precio que, dentro del diferencial de precios comprador-vendedor, sea el más representativo del valor razonable. La IFRS 13 no impide utilizar precios de mercado medios dentro del diferencial comprador-vendedor, siempre y cuando no contradiga este principio.

Por lo general para posiciones largas se utilizan precios de compra (bid) y para posiciones cortas se utilizan precios de venta (ask), porque son usualmente más representativos del valor razonable que usar precios de mercado medios.

\section{FVA - funding value adjustements}

Para ejemplificar el ajuste por financiación y el debate que hay alrededor su estimación, suponga la siguiente transacción:

Las empresas ABC y XYZ negocian un IRS no garantizado (es decir, sin colateral). El valor razonable, excluyendo FVA fue valorado en
500 millones como pérdida no realizada desde la perspectiva de $\mathrm{ABC}$ (es decir, el derivado fue reconocido en el balance de $\mathrm{ABC}$ como pasivo). Como resultado, si $\mathrm{ABC}$ se vuelve insolvente, XYZ tendría que sufrir 500 millones de pérdidas. Análogamente, suponga que XYZ cubre su exposición a riesgo de mercado negociando en posición espejo, un derivado que iguala los términos del derivado original, pero con Bancobertura. Sin embargo, con este sí acordó una garantía (colateral) en efectivo. Como resultado, XYZ tuvo que destinar, suponga 500 millones de efectivo colateral, para mitigar la exposición que Bancobertura tiene frente a XYZ, incurriendo de esta forma, en un costo de fondeo derivado de la financiación de tales garantías en efectivo.

Alternativamente, como el derivado entre ABC y XYZ mostraba una pérdida no realizada de 500 millones, Bancobertura puso, suponga 10 millones de efectivo colateral con XYZ.

En este caso, XYZ participó de dos beneficios relacionados con el colateral recibido:

- Pudo haber ganado un componente de interés por el efectivo recibido en garantía.

- Pudo haber reducido sus necesidades de fondeo frente a Bancobertura.

Por consiguiente, cuando XYZ valoró su derivado con $\mathrm{ABC}$, el día de la negociación, debió tener en cuenta los potenciales costos de fondeo que originan los movimientos favorables en el valor razonable del derivado. Adicional y congruente con esta afirmación, XYZ tuvo que incorporar como ajuste al precio, los potenciales beneficios de fondeo de los futuros movimientos desfavorables en el valor razonable del 
derivado. El ajuste neto es denominado funding value adjustment o ajuste en valoración por fondeo.

Esto parece ir en contravía con la IFRS 13, pues los costos de fondeo son específicos de cada entidad (se podría asimilar a un costo de transacción); entonces, no deberían ser tenidos en cuenta para la valoración. Debido a su subjetividad, no hay convenios de mercado en relación con estos costos.

¿Deberían entonces, dos entidades con diferentes costos de fondeo, tener diferentes valores razonables para un mismo activo? La respuesta de cara a la IFRS 13 es no.

Independientemente de los costos de fondeo, suponga para la entidad A $1 \%$ y para la entidad B 2\% (probablemente, puedan influir en la decisión de compra), pero A y B deben acordar que el valor razonable de la inversión es el valor de mercado. Este valor razonable puede diferir del precio de venta o de publicación que A o B pueden poner a su instrumento financiero, debido, por ejemplo, al costo de fondeo $u$ otras posibles razones.

No se debe confundir valor neto de realización (precio final de venta) con valor razonable (valor de mercado). El valor razonable (y los derivados no son la excepción) es el precio que suple la oferta y la demanda. Es posible que aquellos participantes de mercado que quieren comprar el instrumento financiero, establezcan un precio superior al de mercado, y aquellos que quieran vender, un precio inferior al de mercado.

John Hull y Alan White (2014, p. 16) establecen:
Some argue that funding costs have moved markets away from the "law of one price." We do not agree. Only one price clears the market for any given product. One of the features of derivatives (and of many other financial products) is that they can easily be both bought and sold. If the price is different in two markets, they will be bought in one market and sold in the other, which is why accountants and derivatives dealers should value derivatives at market price, not at cost. Indeed, we have shown how end users and dealers can arbitrage FVA very simply. [Algunos argumentan que los costos de financiamiento han alejado a los mercados de la "ley de un mismo precio". No estamos de acuerdo. Solamente un precio despeja el mercado para cualquier producto dado. Una de las características de los derivados (y de muchos otros productos financieros) es que pueden ser fácilmente comprados y vendidos. Si el precio es diferente en dos mercados, se comprarán en un mercado y se venderán en el otro, por lo que los contables y los negociadores de derivados deben valorar los derivados a precio de mercado, no al costo. De hecho, hemos demostrado que los usuarios finales y los distribuidores pueden arbitrar el FVA de manera muy sencilla].

Una discusión similar surge cuando se añaden los ajustes por riesgo de contraparte y propio CVA/DVA. A pesar de que el proceso valorativo sigue la misma metodología, las contrapartes son diferentes en cuanto a riesgo de crédito se refiere, pues tienen DVA y CVA diferentes. Aunque este caso también podría 
parecer una violación a los principios contables establecidos en la medición a valor razonable de la NIIF 13 (porque por ejemplo, si una parte nova el contrato derivado con una contraparte diferente, el precio al cual se hace la transacción refleja la calidad crediticia de la contraparte).

Sin embargo, cuando se analiza la transacción desde el punto de vista de cada uno de los participantes de mercado, se puede concluir que no hay tal violación y el cálculo del CVA/ DVA tiene lógica económica y debería formar parte del valor razonable del derivado.

Por ejemplo:

Miremos una transacción entre A y B. Desde la perspectiva de A, el valor razonable riskfree del derivado es 100, CVA es 5 y DVA de 10. El valor razonable neto para A es de $105^{5}$ y el valor razonable neto para $\mathrm{B}$ es de $-105^{6}$.

Ahora, supongamos que A nova el derivado con Bancobertura, una empresa sin riesgo de crédito. Suponga que el riesgo de crédito de Bancobertura es 0 , así que el valor razonable ahora es 957. Si C cierra la transacción con A pagando 95, el valor para Bancobertura es 95 y el valor para B es -95 .

Aparentemente, 10 fueron pérdidas asumidas por A. Sin embargo, B estaría dispuesto a monetizar los 10 de ganancia por concepto de DVA para facilitar la novación del derivado hacia Bancobertura.

Asumiendo que este pago sucede, A recibiría 95 de Bancobertura y 10 de B. B pagaría 10 a A y terminaría con una transacción que vale

$5105=($ Risk-free $)$ 100-(CVA) 5+ (DVA) 10.

$6-105=($ Risk-free $)-100-($ CVA $) 10+($ DVA $) 5$.

$795=($ Risk-free $) 100-(5) 0+($ DVA $) 0$
-95. En otras palabras, la valoración original de 105 para A y de -105 para B, permanece.

El ejemplo anterior pone de manifiesto otra de las críticas que sostienen algunos autores (aparte de la supuesta contraintuitividad) del modelo, y es que se están reflejando en el resultado ganancias (pérdidas) que no serían realizables al momento de su medición del valor razonable como exit price. Sin embargo, la lógica económica y la práctica de mercado están detrás del ajuste contable.

Suponga que Pepo tiene a su nombre un seguro de vida por 1 billón. Suponga también, que Pepo sube al último piso del edificio Colpatria y amenaza con lanzarse al vacío. Llama a su compañía aseguradora ofreciéndole monetizar el contrato a cambio, digamos del 50\% (0,5b) de su valor. Como beneficio, la aseguradora no tendrá que responder en caso de muerte real por el nominal asegurado.

Si la compañía de seguros cree en el intento de suicidio de Pepo, estaría dispuesta a pagar el monto que exige el asegurado (suponga que el costo de oportunidad de pagar la mitad de la póliza supera el hecho de pagar todo al final).

Este ejemplo no es irracional y sucede día a día en las transacciones con derivados. Las terminaciones antes del vencimiento (unwinds) o como se observó en el ejemplo anterior con las novaciones (sustituir contrapartes de derivados), son regulares en los mercados financieros.

Suponiendo una transacción donde CVA y DVA son iguales, por ejemplo 100 y (100), ante el deterioro de la calidad crediticia de una de las partes, por ejemplo CVA es (300) pero la calidad crediticia de la contraparte permanece en 100, cerrar la transacción de forma anticipa- 
da es siempre una opción realista pagando una prima (monetizando el DVA) por 200. Como se demostró en el ejemplo anterior, la novación funciona de forma similar. Sustituir la contraparte por una con mejor calidad crediticia para que el derivado continúe valiendo 100, costaría una prima de 200 (monetizando el DVA).

Sin embargo, aunque hay un interés económico que los participantes de mercado estarían dispuestos a asumir, regularmente la monetización no se da de forma perfecta.

Jon Gregory (2012) lo expone así:

There is evidence in OTC derivatives markets of institutions being able to monetise DVA via unwinds and novations. However, there is a problem making this monetisation imperfect. The conterparty, knowing the institution is making a gain via DVA, may wish to profit themselves. They will therefore not be willing to unwind but a lower value, being the obvious value (split the difference). In such case, only half of the DVA has been monetised.

[Hay evidencia en los mercados de derivados OTC que las instituciones son capaces de monetizar DVA por medio de unwinds y novaciones. Sin embargo, hay un problema que hace que esta monetización sea imperfecta. La contraparte, sabiendo que la institución está obteniendo beneficios por medio de DVA, puede desear beneficiarse. Por lo tanto, puede no estar dispuesta a realizar un unwind a un valor menor, siendo el valor obvio (dividir la diferencia). En ese caso, solo la mitad de la DVA ha sido monetizada].
No obstante, contrario a lo que pasa con el CVA y DVA, el problema que subyace al FVA, es que los participantes de mercado pueden tener diferentes valores razonables incluso utilizando los mismos modelos de valoración. Suponiendo que la variable de costos de fondeo sea la misma o haya una convención de mercado, el FVA (y por consiguiente el valor razonable) del comprador sería igual en magnitud y signo al FVA de la contraparte. Sin embargo, en la práctica esto no sucede, comprador y vendedor tienen diferentes costos y formas de fondeo; por consiguiente, llegarán a valores razonables diferentes.

Una de las posibles consecuencias de continuar midiendo valores razonables incluyendo costos de fondeo es puesta en evidencia por John Hull y Alan White (2014):

A highly creditworthy end user can buy options from a bank with high funding costs and sell the options to a bank with low funding costs in such a way that all three parties appear to be making a profit. The possibility of accounting systems being gamed in this way will clearly not be tolerated. There is also a danger that traders will use FVA to inflate their profits on long-dated deals in which options or similar derivatives are sold. (The scope for inflating profits increases with the life of the underlying option.) [Un usuario final altamente solvente puede comprar opciones de un banco con altos costos de financiamiento y vender las opciones a un banco con bajos costos de financiamiento de tal manera que las tres partes parecen estar obteniendo ganancias. La posibilidad 
de que los sistemas de contabilidad sean manipulados de esta manera claramente no se tolerará. También existe el peligro de que los comerciantes utilicen la FVA para inflar sus ganancias en contratos de larga duración en los que se vendan opciones o derivados similares. (El margen para aumentar los beneficios aumenta con la vida de la opción subyacente)].

\subsection{Ejemplo de cálculo de riesgo} de contraparte para un derivado y su sentido económico en el balance

El reto financiero que enfrenta la contabilidad radica en cómo reflejar en un ajuste contable, la exposición presente de un derivado OTC, cuyos flujos de efectivo futuros son inciertos (dependen de un subyacente) y traducir esto cuantitativamente en cada una de las fechas de balance.

Con el fin de ejemplificar cómo se calcularía y analizar su relación económica e interpretación, sigamos este ejemplo:

El 01 julio de 2010, ABC adquirió un pasivo US\$500 millones atado a la DTF 6 meses. Con el fin de cubrir el interés, entró en un IRS pa- ga-fijo recibe-variable con Bancobertura (swap no colateralizado) en el cual paga un tipo fijo del 3,2\% y recibe DTF sobre un nocional de US\$500 millones.

Los términos del IRS se resumen a continuación:

\begin{tabular}{ll}
\hline Fecha de negociación & 01-jul-10 \\
Contraparte & ABC y Bancobertura \\
Nocional & US\$500 millones \\
Fecha de liquidación & 30 -jun-15 \\
ABC paga & $2,20 \% /$ base 30/360 \\
ABC recibe & DTF 6 meses / base 30/360 \\
Períodos de interés & Cada 6 meses \\
\hline
\end{tabular}

El 1 de julio de 2013 (2 años antes de cumplimiento), ABC revaluó el activo. A esa fecha, los precios comprador-vendedor en tipos de interés de referencia estaban en 3,41\% y ABC estimó con base en datos observables de mercado, que el precio bid sería de 1 punto básico $(0,01 \%)$ resultando en una tasa de un swap a dos años de 3,40\%. La valoración risk-free del derivado, antes de ajustes por riesgo de crédito de contraparte CVA-DVA o FVA, era US\$1.494 millones calculado de la siguiente forma:

\begin{tabular}{lccccccc}
\hline Liquidaciones & $\begin{array}{c}\text { DTF 6 } \\
\text { meses }\end{array}$ & $\begin{array}{c}\text { Fixed } \\
\text { rate }\end{array}$ & $\begin{array}{c}\text { Monto } \\
\text { previsto } \\
\text { floating } \\
\text { leg }\end{array}$ & $\begin{array}{c}\text { Monto } \\
\text { previsto } \\
\text { fixed } \\
\text { leg }\end{array}$ & $\begin{array}{c}\text { Monto } \\
\text { previsto } \\
\text { neto }\end{array}$ & $\begin{array}{c}\text { Factor de } \\
\text { descuento }\end{array}$ & $\begin{array}{c}\text { Valor } \\
\text { presente } \\
\text { monto } \\
\text { esperado }\end{array}$ \\
\hline 31-dic & $2,0 \%$ & $3,2 \%$ & 5.000 & 8.000 & -3.000 & $99,0 \%$ & $-2.970,30$ \\
30-jun & $3,0 \%$ & $3,2 \%$ & 7.500 & 8.000 & -500 & $97,5 \%$ & $-487,73$ \\
31-dic & $4,0 \%$ & $3,2 \%$ & 10.000 & 8.000 & 2.000 & $95,6 \%$ & $1.912,68$ \\
30-jun & $4,5 \%$ & $3,2 \%$ & 11.250 & 8.000 & 3.250 & $93,5 \%$ & $3.039,71$ \\
\hline TOTAL & & & & & & & $1.494,36$ \\
\hline
\end{tabular}

Tabla 1

Fuente: elaboración propia 
Los dos montos de liquidación negativos de $(2.970,3)$ y $(487,73)$ representan importes que $\mathrm{ABC}$ tendría que pagar en cada una de las liquidaciones (el swap se va "desinflando" con el tiempo). La lógica económica de esto es que en esas fechas, Bancobertura estará expuesto al riesgo de crédito de la empresa $\mathrm{ABC}$. Por el contrario, los montos de liquidación positivos, 1.912,68 y 3.039,71 en los dos últimos períodos, respectivamente, significan que $A B C$ estaría expuesto al riesgo de contraparte de Bancobertura.

\section{Paso 1. División de la exposición en períodos}

Para calcular el riesgo de crédito, lo primero que debe hacerse es dividir el tiempo de vida del derivado en períodos (para el swap, sería apropiado dividirlo en semestres pues son los tiempos en los cuales hay exposición a riesgo de crédito de contraparte y propio). En caso de que no haya liquidaciones periódicas como puede ser el caso de otros derivados, la subdivisión puede ser política de la entidad bajo un racio- nal establecido. Por ejemplo, dependiendo de la variabilidad el subyacente o de thresholds, entre otros.

Para un swap, coincidir las fechas de liquidación con las fechas de cálculo del CVA es apropiado, debido a la exposición a los recibos/ pagos monetarios.

\section{Paso 2. Cálculo de la exposición al momento de default (Exposure at default, AED)}

El segundo paso es calcular el valor presente de la AED para cada período. La exposición al momento de default representa la valoración risk-free del swap (o derivado) a cada momento. O, en otras palabras, la exposición de ambas contrapartes al default en cada momento. Una forma sencilla es suponer que el subyacente se comportará como espera el mercado (el tipo de interés en nuestro caso se comportará como se presupuesta).

La exposición esperada en el período 1 se calcula a partir de la valoración risk-free justo antes la liquidación de los (3.000) así:

\section{Exposición esperada período 1}

\begin{tabular}{lcccc}
\hline Liquidaciones & $\begin{array}{c}\text { DTF 6 } \\
\text { meses }\end{array}$ & $\begin{array}{c}\text { Factor de } \\
\text { descuento }\end{array}$ & $\begin{array}{c}\text { Monto } \\
\text { previsto neto }\end{array}$ & $\begin{array}{c}\text { Valor presente monto } \\
\text { esperado }\end{array}$ \\
\hline 31-dic & $2,0 \%$ & 1,00 & -3.000 & -3.000 \\
30 -jun & $3,0 \%$ & 0,99 & -500 & -493 \\
31 -dic & $4,0 \%$ & 0,97 & 2.000 & 1.932 \\
30 -jun & $4,5 \%$ & 0,94 & 3.250 & 3.070 \\
\hline TOTAL & & & & 1.509 \\
\hline
\end{tabular}

Tabla 2

Fuente: elaboración propia 
La exposición esperada en el período 2 se calcula como la exposición al final del período 1 menos (3.000). La exposición final del período
2 corresponde a la valoración risk-free justo antes de la liquidación de los (493):

Exposición esperada período 2

\begin{tabular}{lcccc}
\hline Liquidaciones & $\begin{array}{c}\text { DTF 6 } \\
\text { meses }\end{array}$ & $\begin{array}{c}\text { Factor de } \\
\text { descuento }\end{array}$ & Monto previsto neto & $\begin{array}{c}\text { Valor presente monto } \\
\text { esperado }\end{array}$ \\
\hline 30 -jun & $3,0 \%$ & 1,00 & -500 & -500 \\
31 -dic & $4,0 \%$ & 0,98 & 2.000 & 1.961 \\
30 -jun & $4,5 \%$ & 0,96 & 3.250 & 3.116 \\
\hline TOTAL & & & 4.577 \\
\hline
\end{tabular}

Tabla 3

Fuente: elaboración propia

Las exposiciones de los períodos 3 y 4 son replicadas de acuerdo con las tablas anteriores y se omiten para simplificar los cálculos.
La tabla 4 resume el valor presente de la EAD para cada exposición esperada en cada momento del derivado:

\begin{tabular}{cccc}
\hline $\begin{array}{c}\text { Exposición esperada } \\
\text { \# período }\end{array}$ & $\begin{array}{c}\text { Exposición } \\
\text { esperada inicial }\end{array}$ & $\begin{array}{c}\text { Exposición } \\
\text { esperada final }\end{array}$ & Promedio EAD \\
\hline 1 & 1.494 & 1.509 & $1.501,83$ \\
2 & 4.509 & 4.577 & $4.543,13$ \\
3 & 5.077 & 5.178 & $5.127,71$ \\
4 & 3.178 & 3.250 & $3.214,24$ \\
\hline
\end{tabular}

Tabla 4

Fuente: elaboración propia

\section{Paso 3. Cálculo de la probabilidad al momento de default (PD) y la pérdida dado el incumplimiento (LGD)}

El tercer paso en el cálculo de riesgo de contraparte consiste en calcular la probabilidad de default (PD). Como se muestra en la tabla 4, la exposición al momento de default da positiva. En otras palabras, $\mathrm{ABC}$ está expuesta al riesgo de crédito de contraparte de Bancobertura.

La probabilidad al momento de default puede ser calculada si Bancobertura tiene deuda cotizada (como referencia en comparación a los tipos de interés libres de riesgo) o si tiene CDS. Otra forma práctica de hacerlo es tomando como referencia su calificación crediticia y utilizar matrices que asignen ratings a PD en diferentes momentos.

Suponiendo que la PD de Bancobertura para cada período de análisis es calculada a partir de cualquiera de los anteriores métodos (por ejemplo, probabilidad de default a partir de rating), se tiene la tabla 5: 


\begin{tabular}{ccc}
\hline $\begin{array}{c}\text { \# } \\
\text { Liquidaciones }\end{array}$ & $\begin{array}{c}\text { Años (coinciden } \\
\text { con liquidación } \\
\text { del swap) }\end{array}$ & PD \\
\hline 1 & 0,5 & $0,30 \%$ \\
2 & 1,0 & $0,40 \%$ \\
3 & 1,5 & $0,45 \%$ \\
4 & 2,0 & $0,50 \%$ \\
\hline
\end{tabular}

Tabla 5

Fuente: elaboración propia
La pérdida dado el incumplimiento por lo general se asume que es de un $40 \%$ a un $60 \%$. En caso de incumplimiento, $\mathrm{ABC}$ no perderá todo debido a posibles acuerdos que mitiguen el riesgo o a partir de la prelación de pago a los acreedores. Asumamos que el de Bancobertura es 40\%.

Con base en los cálculos anteriores, el CVA para cierto EAD puede ser calculado como el valor presente de la pérdida esperada futura así:

\begin{tabular}{ccccc}
\hline \# Liquidaciones & PD & LGD & VA exposición & CVA por liquidación \\
\hline 1 & $0,30 \%$ & $40 \%$ & $1.501,83$ & 1,80 \\
2 & $0,40 \%$ & $40 \%$ & $4.543,13$ & 7,27 \\
3 & $0,45 \%$ & $40 \%$ & $5.127,71$ & 9,23 \\
4 & $0,50 \%$ & $40 \%$ & $3.214,24$ & 6,43 \\
\hline TOTAL & & & & 24,73 \\
\hline
\end{tabular}

Tabla 6

Fuente: elaboración propia

El CVA total es de 24,73 millones correspondiente a aproximadamente un $2 \%$ de la valoración risk-free del swap.

\section{Debit value adjustment}

En el ejemplo anterior, EAD era positivo, lo que significa que el riesgo de crédito de contraparte esperado durante toda la vida del swap era una exposición constante al riesgo de crédito de Bancobertura. Por el contrario, Bancobertura nunca estuvo expuesto al riesgo de crédito de ABC. Sin embargo, puede haber situaciones en las cuales estén presentes ambos. EAD positiva (sujeto a la PD de Bancobertura) y EAD negativos (sujeto a la PD de ABC).

\section{Dificultades prácticas en la aplicación del modelo}

La modelación de las pérdidas por riesgo de crédito y otros ajustes pueden llegar a ser complejos, dependiendo del tipo de entidad que los calcule, su infraestructura y capacidad tecnológica y de la naturaleza misma de los derivados. Por lo general, el ajuste no debería ser material entre contrapartes que comparten similares riesgos de crédito (nótese que se estaría multiplicando una PD de diferente signo por una exposición igual - o constante-). Sin embargo, en entidades que están expuestas a diversos riesgos y que negocian derivados a largo plazo, pueden tener un ajuste importante en resultados por valoración. 
Hay otras formas de calcular los ajustes por riesgo de crédito de contraparte, unas más precisas que otras y algunas que no deberían ser aceptadas.

En nuestro ejemplo, la exposición al momento de default (AED) para cada período de tiempo asume que los tipos de interés durante la vida del instrumento financiero se comportarán como lo esperado. Sin embargo, en la práctica, no es tan cierto que los subyacentes se muevan de forma predecible. Un modelo más realista debería incluir una simulación de los factores de mercado. Esto supondría en nuestro ejemplo, que la DTF (subyacente) pueda comportarse de diversas formas a través de los años de la vida del derivado. Esta simulación normalmente es llevada a cabo usando el método Monte Carlo, con el cual se pueden simular mediante procesos estocásticos cientos de potenciales caminos en los movimientos de los factores de mercado. Este es el proceso más complejo en la simulación, en especial cuando hay diversos factores de mercado que pueden afectar la exposición futura del derivado (imagínese la simulación para una basket o spread option).

Ahora bien, hay opciones intermedias al cálculo de la AED (para no calcular el valor del derivado muchas veces), por ejemplo, mediante Add-on(s), en que se simulan típicamente dos escenarios (por ejemplo, uno positivo y otro negativo) adicionando o disminuyendo la exposición del derivado presente, teniendo en cuenta componentes que representan la incertidumbre de la EAD futura (por ejemplo, esta forma hace parte de las reglas de capital expuestas en el Acuerdo de Basilea I, de 1988).

\section{Conclusiones y reflexión}

Las NIIF están planteando una serie de desafíos interdisciplinarios que deben ser tenidos en cuenta. Las transacciones complejas de mercado provocan un impacto en la representatividad de riesgos y rendimientos que las transacciones tienen para los balances de las entidades.

Infortunadamente, algunos organismos que supervisan, vigilan y controlan se limitan a modificar las normas para incluir excepciones y tercerizar procesos sin tener en cuenta los efectos colaterales que sobre la práctica contable y sobre la interpretación de cifras en balance, tiene la introducción en el mercado de estas reformas.

Si bien en algunos casos podría llegar a sustentarse la exclusión de ciertos requerimientos, muchos de ellos corresponden a intereses diferentes de los económicos y los que la disciplina persigue. No es justificable la aparente "dificultad" en la contabilización para una profesión que es económica y financiera. Tampoco es justificación no hacerlo apoyándose en un criterio deliberado de "materialidad" utilizando un juicio aprovechado.

Tal como lo expuso FASB, si los valores razonables no cumplen las características de la NIIF 13, no son valores razonables e irían en contravía de las características cualitativas de la información financiera. Bajo ninguna lógica serían estados financieros que "cumplan con las NIIF" y mucho menos, auditados sin salvedades por revisor fiscal.

Infortunadamente, en muchos casos se trata de un trasfondo académico y de continua actualización (competencia) profesional que cultu- 
ralmente la profesión tiende a evadir. Muchos prefieren modificar las normas a reaprender. El mundo detrás de las NIIF enseña que lo que hasta ahora se conoce no es útil en un proceso de representación de la realidad de los mercados. Los modelos actuales son incapaces de reflejar las transacciones complejas en las cuales un sinnúmero de factores afectan los balances y por consiguiente, la toma de decisiones en las entidades.

Ahora bien, unido con lo anterior se encuentra la utilización deliberada y excesiva de la proveeduría de precios (tercerización). Los ajustes a valor razonable que impactan el patrimonio de las entidades vía utilidades retenidas $\mathrm{u}$ otros resultados integrales, deben ser de entero conocimiento financiero y análisis cuantitativo por la gerencia de la entidad.

La NIIF 13.B46 lo establece así (itálicas añadidas):

(...) una entidad evaluará si los precios estimados proporcionados por terceros están desarrollados utilizando información actual que refleje transacciones ordenadas o una técnica de valoración que refleje los supuestos de los participantes del mercado (incluyendo supuestos sobre el riesgo). Al ponderar un precio estimado como un dato de entrada en una medición del valor razonable, una entidad dará menos peso (en comparación con otros indicios del valor razonable que reflejen los resultados de las transacciones) a estimaciones que no reflejan el resultado de transacciones.
¿Cuál es el motivo de querer excluir a los contadores de ciertos cálculos? ¿Será algo que motivó la propia profesión? ¿Será autoimpuesto? ¿O el mercado (el regulador) no piensa que los profesionales son lo suficientemente idóneos y por ello, terceriza?

Burzum (citado por IASB, Expert Advisory Panel, 2009) explica:

Así ocurrió desde el invento, en el mundo, de la primera calculadora mecánica que Blaise Pascal (1623-1662) creó para que la usaran los contadores del tribunal de cuentas que al servicio de Richelieu dirigía su padre. Ello estuvo asociado con el primer intento de producción en serie de 50 de estas máquinas calculadoras, intento que fracasó por falta de mecánicos competentes, elevados costos de producción (Kung, 1979, p. 27) pero sobre todo porque "los contadores se negaron a usarlas, aferrados a la pluma y a la tinta”.

La defensa de las prácticas ancestrales y el "efecto reiteración" deben ser abolidos en el proceso de convergencia como beneficio añadido a la profesión. Volver a las prácticas del pasado no puede ser la opción. No se puede seguir pagando altos costos por la pasividad y la oposición que permiten que surjan y se consoliden otras profesiones no contables en un mundo que debería ser exclusivo de la profesión.

Hoy, valuadores, economistas, ingenieros, matemáticos y financieros han tomado las riendas de elaborar, explicar e interpretar cifras de balance. Y lo peor de todo, los contadores deben trabajar subordinados a lo que diga "el experto" por querer continuar quedándose 
en el pasado sin querer apropiarse de la dinámica que impone el mercado actual. Para la desafortunada pasividad, el mundo continúa su curso y las transacciones siguen evolucionando.

Los contadores tienen que adaptarse a la dinámica expuesta. Los problemas deben solucionarse en el presente y para el futuro, antes que recurrir a descargar responsabilidades sobre otras profesiones.

Conforme pasan los meses, veo con descontento cómo se incrementan las excepciones y trabas a la aplicación de las IFRS. ¿No se supone que la Ley 1314 de 2009 pretende todo lo contario? (¿Estamos en un proceso de desconvergencia?). El continuo establecimiento de brechas entre las NIIF y lo local va en evidente contravía de la construcción de un sistema único del que habla la ley.

El gobierno continúa delegando funciones en organismos como si la solución a todo fuese la descentralización. Parece que es de su interés escuchar lobistas y hacer caso a minorías sin bases técnicas, pero motivados ambos por intereses diferentes a los que persigue la profesión. No podemos volver al pasado y la contabilidad no puede ser vista como una herramienta que apoye la corrupción financiera. No puede haber interferencia política en la emisión de estándares de contabilidad.

\section{Referencias}

Colombia (2009). Ley 1314 de 2009, por la cual se regulan los principios y normas de contabilidad e información financiera y de aseguramiento de información aceptados en Colombia, se señalan las autoridades competentes, el procedimiento para su expedición y se determinan las entidades responsables de vigilar su cumplimiento, Diario Oficial, 47.409, 13 de julio de 2009. Disponible en: http://www. secretariasenado.gov.co/senado/basedoc/ ley_1314_2009.html

Comité de Supervisión Bancaria de Basilea (2001). El Nuevo Acuerdo de Capital de Basilea. Disponible en: http://www.bis.org/ publ/bcbsca03_s.pdf

Financial Accounting Standard Board, FASB (2006). Fair Value Measurements. Statement of Financial Accounting Standards 157. Disponible en: http://www. fasb.org/cs/BlobServer?blobcol=urldata\&bl obtable $=$ MungoBlobs\&blobkey=id\&blobw here $=1175820927537 \&$ blobheader $=$ applic ation\%2Fpdf

Gregory, Jon (2012). Counterparty Credit Risk: A Continuing Challenge for Global Financial Markets. London: John Wiley \& Sons Ltd. Hull, John \& White, Alan (2014). Valuing Derivatives: Funding Value Adjustments and Fair Value. Financial Analysts Journal, 70 (3), 46-56. Disponible en: http://www-2.rotman.utoronto. $\mathrm{ca} / \sim$ hull/downloadablepublications/ FVAandFairValue.pdf International Accounting Standard Board, IASB (June 2009). Credit Risk in Liability Measurement. Disponible en: http://www.ifrs. org/Current-Projects/IASB-Projects/CreditRisk-in-Liability-Measurement/DP-Jun-09/ Documents/CreditRiskLiabilitStaff.pdf International Accounting Standards Board, IASB (2011). IFRS 13: Fair Value 
Measurement. Disponible en: http://www. ifrs.org/Current-Projects/IASB-Projects/ Fair-Value-Measurement/IFRS-13-FairValue-Measurement/Pages/IFRS-13-FairValue-Measurement.aspx

International Accounting Standards Board, IASB, Expert Advisory Panel (2009). Medición y revelación del valor razonable de instrumentos financieros en mercados que ya no están activos. En Samuel Alberto Mantilla (comp., trad.). Contabilidad a valor razonable: en el contexto de la crisis actual de los mercados, 133-218. Bogotá: ECOE Ediciones.

Martínez-Pineda, Pablo Enrique (2010). Una dificultad inherente de la profesión analizada desde el punto de vista de la medición. Revista Estudiantil Identidad Contable, 1 (1), 77-89. Merton, Robert C. (1974). On the Pricing of Corporate Debt: The Risk Structure of Interest Rates. Journal of Finance, 29 (2), 449-470. Morales-Díaz, José (2010). Pasivos financieros a valor razonable: la consideración del propio riesgo de crédito. Foro AECA Instrumentos Financieros (FAIF). Disponible en: http:// aeca.es/old/faif/articulos/comunicacion5.pdf Ramírez, Juan (2015). Accounting for Derivatives: Advanced Hedging under IFRS 9. London: John Wiley \& Sons Ltd.
Ryan, Stephen (2008). Fair Value Accounting: Understanding the Issues Raised by the Credit Crunch. Disponible en: http://www.cii.org/ files/publications/white_papers/07_11_08_ fair_value_accounting.pdf

United States, Security and Exchange Commission, SEC, Office of the Chief Accountant, Division of Corporation Finance (2008). Report and Recommendations Pursuant to Section 133 of the Emergency Economic Stabilizing Act of 2008: Study on Mark-to-Market Accounting. Disponible en: https://www.sec.gov/files/ marktomarket123008.pdf

- Fecha de recepción: 2 de marzo de 2016

- Fecha de aceptación: 22 de julio de 2016

- Disponible en línea: 14 de diciembre de 2016

\begin{tabular}{|l|}
\hline Para citar este artículo \\
Martínez, Pablo (2016). Credit, debit \& fun- \\
ding value adjustments en valoración de \\
instrumentos financieros: la realidad eco- \\
nómica detrás del ajuste contable. Cua- \\
dernos de Contabilidad, 17 (44), 521-543. \\
https://doi.org/10.11144/Javeriana.cc17- \\
44.cdf
\end{tabular}


